Article

\title{
Preparation of Electrosprayed Poly(caprolactone) Microparticles Based on Green Solvents and Related Investigations on the Effects of Solution Properties as Well as Operating Parameters
}

\author{
Shengchang Zhang *, Christine Campagne and Fabien Salaün \\ GEMTEX-Laboratoire de Génie et Matériaux Textiles, ENSAIT, F-59000 Lille, France; \\ christine.campagne@ensait.fr (C.C.); fabien.salaun@ensait.fr (F.S.) \\ * Correspondence: shengchang.zhang@ensait.fr; Tel.: +33-633-703-189
}

Received: 11 December 2018; Accepted: 29 January 2019; Published: 30 January 2019

\begin{abstract}
Electrosprayed poly(caprolactone) (PCL) microparticles were produced using five solvents (ethyl acetate, acetone, anisole, glacial acetic acid and chloroform) under different PCL concentrations and operating parameters. Not only green and appropriate solvent for PCL electrospraying was pointed out, but also the effects of solution properties (surface tension, electrical conductivity, viscosity and vapor pressure) and operating parameters (flow rate, working distance and applied voltage) on the formation of electrosprayed particles were clarified. The formation and shape of Taylor cone during electrospraying was observed by high-speed images captured with a camera, and the size and morphology of electrosprayed particles were characterized by optical and scanning electron microscopies. It can conclude that the cone-jet range of applied voltage mainly depended on electrical conductivity, and an ideal Taylor cone was easier to form under high viscosity and low surface tension. Although high electrical conductivity was a contributor to fabricate tiny particles, it was easier to fabricate mono-dispersed microparticles under low electrical conductivity. The poly-dispersed distribution obtained with a high electrical conductivity converted into mono-dispersed distribution with the increasing of viscosity. Furthermore, the size of electrosprayed particles also correlated with the surface tension and vapor pressure of the solvent used. Ethyl acetate, due to mild electrical conductivity and surface tension, moderate viscosity and vapor pressure, is a green and suitable solvent for PCL electrospraying. Single pore PCL microparticles with smooth cherry-like morphology can be prepared from ethyl acetate. Finally, long working distance not only stabilizes the break-up of charged jet, but also promotes the evaporation of solvent.
\end{abstract}

Keywords: poly(caprolactone) microparticles; electrospraying; solvent properties; operating parameters; Taylor cone; size and morphology

\section{Introduction}

For the last few decades, electrospraying technique, as a novel electrohydrodynamic atomization process, has been widely used to prepare polymeric microspheres or microcapsules as well as further achieve their functional coatings on materials surface [1-5]. Compared with other common technologies to fabricate particles in the micro- or nanoscale from polymeric solution, including an emulsification method, spray drying, complex coacervation, self-assembly and flashing nanoprecipitation [6-10], electrospraying allows for overcoming the main drawbacks and limits them. For example, the agglomeration of particles, wide particles size distribution and low encapsulation efficiency to active principle as main limitations coming from these technologies can be improved effectively in electrospraying process via the Coulombic repulsion among charged droplets and using 
coaxial nozzle. The particle size distribution of electrosprayed particles is mono-dispersed under optimal conditions, and the morphological and structural design of electrosprayed microparticles can carry out effectively via changing solution properties and operating parameters. Meanwhile, the use of surfactant and intensive stirring in emulsification method, which may break down the structure and properties of microcapsules, is avoided in the electrospraying process. The complete entrapment of active principle from electrospraying allows for protecting it at maximum based on experimental conditions. In addition, being different from complex coacervation, self-assembly and the emulsification method, the experimental setup and operations of electrospraying are easy to implement (usually being done in one step), and the emission of toxic substance and environmental pollution during electrospraying can be limited to the minimum. Although flashing nanoprecipitation as a solvent displacement technology also obtains polymeric particles easier and rapidly, the high level of requirements in the state of working streams (strong turbulence), special micro-mixers and the lipophilicity of active substances make it less feasible compared with electrospraying. Therefore, electrospraying is regarded as a green, rapid and efficient technology to design polymeric microparticles.

In the electrospraying process, the formation of a Taylor cone is a required step to ensure the preparation of polymeric particles having a tiny size and a narrow size distribution. Meanwhile, the effective control on the structure and morphology of electrosprayed particles can be only carried out under cone-jet mode [11-13]. Actually, the formation of Taylor cone is mainly governed by several forces when the solution flows out from the electrified nozzle, i.e., (i) gravity of liquid, (ii) surface tension of droplet, (iii) electrostatic attraction, (iv) viscoelastic force of fluid, (v) Coulomb repulsion among charged liquid, and (vi) friction between droplets and air [14,15]. Once these forces reach a certain level at the tip of the nozzle, charged droplets are stretched into conical shape and then accelerated into the charged jet. Then, it breaks up into tiny and uniform droplets due to the presence of Coulomb repulsion. Finally, solvent evaporation occurs during the flight process to lead to the formation of solid particles. Therefore, electrosprayed particle size characteristics, as well as their morphologies, also depend on the break-up process of charged jet and the evaporation of solvent. The formation of the Taylor cone is significantly affected by the solution properties (surface tension, electrical conductivity, viscosity and vapor pressure) and operating parameters (polymer concentration, flow rate, working distance and applied voltage), like the break-up of charged jet and the evaporation of solvent [16-19]. For instance, as the resistance of the formation of Taylor cone, low surface tension (lower than $50 \mathrm{mN} / \mathrm{m}$ ) makes the stable cone-jet mode more accessible. Thus, under high surface tension, intermittent cone-jet is obtained due to the cone relaxation resulted from space charge. According to Stachewicz et al., the electrospraying of high surface tension liquid (i.e., water) will be carried out under stable cone-jet when using single event electrospraying (SEE) to avoid cone relaxation based on pulse-controlled liquid deposition [20]. Meanwhile, the charge transfer in electrified liquid cones as well as related relationships among solution properties, liquid flow rate, emit currents and jet diameter were closely related to the electrical conductivity of working liquid [21]. In addition, Rosell-Llompart et al. found that the formation of a stable cone-jet mode is obtained under the minimum liquid flow rate [22]. The mean diameter, as well as the size distribution of electrosprayed droplets, mainly depends on flow rate of liquid, electrical conductivity of liquid and viscosity of the liquid. Furthermore, the various resulting shapes of Taylor cones under cone-jet mode also lead to different mean diameters and size distributions of the obtained solid microparticles [23].

Poly(caprolactone) (PCL) as a non-toxic, biodegradable and biocompatible polyester is widely applied in the fields of drug control release and delivery, tissue engineering and functionalization of textile surface [24-27]. PCL microspheres or microcapsules prepared from the electrospraying processs have various advantages and application values in these fields. The requirements concerning controlled release behavior of active substance or degradation rate of the polymer matrix can be adjusted via designing the size and morphology of electrosprayed microcapsules according to the targeted application [28,29]. Meanwhile, for drug delivery efficiency, electrosprayed microcapsules with fine 
size and narrow size distribution have an enhanced ability to reach their target [30]. The existence of a fine core-shell structure allows avoiding the initial burst effect of the active principle, which extends its shelf-life [31]. In addition, the porous morphology obtained from the electrospraying process also improves the specific contact area and interfacial interactions between PCL microparticles and cells for drug control and tissue engineering [32].

Until now, dichloromethane and chloroform have been the main selected solvents to prepare PCL microparticles by electrospraying, but their inherent toxicity and carcinogenicity are harmful to the human body, and limit their further applications in the biomedical field [1,33,34]. Searching for appropriate substitutions of these solvents to carry out PCL electrospraying is imperative for satisfying the requirement of technological development [35]. Non-toxic, green and environment-friendly, but also suitable physico-chemical properties for PCL electrospraying process as well as outcome are important for the choice of the solvent [36,37]. There have been some papers publishing some simple relationships among structure as well as the morphology of electrosprayed particles, solution properties and operating parameters $[1,12,38]$. However, these conditions to determine the formation and shape of Taylor cones are still superficial and unclear. Meanwhile, the suitable operating parameters for achieving cone-jet also strictly depend on the solution properties. Furthermore, as a multi-physical process, the effects coming from operating parameters and solution properties on electrospraying are complex and interactional. The relationships among solution properties, operating parameters and structural characteristics of electrosprayed particles are also different based on different solvent systems. Therefore, the suitable solvent properties as well as operating parameters to develop electrosprayed particles with satisfied size, as well as morphology, also need further investigation. In addition, although some green solvents (i.e., anisole and acetone) have been introduced to prepare PCL microspheres, their applications in PCL electrospraying is still unreported [35,39].

In order to clarify the effects of solvent properties on electrospraying and obtain appropriate green solvent used for PCL electrospraying, the study aimed to prepare PCL microparticles using four green solvents (ethyl acetate, acetone, anisole and glacial acetic acid) according to the direction of REACH (Registration, Evaluation, and Authorization of Chemicals) and the regulation of European Pharmacopoeia (belonging to the Class 3), in order to find the best possible alternative to the chlorinated solvents commonly used in this process $[35,40]$. The influences of the solution properties and operating parameters during the electrospraying process on the formation and shape of Taylor cone, and microparticles characteristics, i.e., mean diameter, particle size distribution and morphology were studied to determine the most suitable solvents (solution properties) and compare to the use of chloroform. High-speed images captured by a camera were used to analyse the formation and shape of Taylor cones under different solvents and operating parameters. The size and morphology of PCL microparticles prepared from different solvents and operating parameters were characterized by optical and scanning electron microscopies. The purpose of this research is to find some relevant relationships among the solvent properties, the operating parameters, the formation and shape of Taylor cones, and the size and morphology of PCL microparticles in order to determine the optimal electrosprayed conditions and obtain the substitution of chloroform.

\section{Materials and Methods}

\subsection{Materials}

Polycaprolactone (PCL) (Capa ${ }^{\mathrm{TM}}$ 6400, mean molecular weight: 37,000 g/mol) was purchased from Perstorp (Skåne County, Sweden). Chloroform (analytical reagent), ethyl acetate (analytical reagent), anisole (analytical reagent), acetone (analytical reagent) and glacial acetic acid (analytical reagent) were purchased from Sigma Aldrich (Dorset, UK). All reagents were used as received without any further treatment. 


\subsection{Preparation of PCL Electrospraying Solutions}

PCL electrospraying solutions were prepared by dissolving appropriate quantities of PCL pellets in each of five solvents (chloroform, ethyl acetate, anisole, acetone, and acetic acid) at $40{ }^{\circ} \mathrm{C}$ with stirring for $1 \mathrm{~h}$ to allow complete dissolution. For each solvent, five PCL concentration ( $1 \mathrm{wt} \%, 3 \mathrm{wt} \%$, $5 \mathrm{wt} \%, 7 \mathrm{wt} \%$ and $10 \mathrm{wt} \%$ ) were prepared. Before electrospraying, each solution was cooled to room temperature $\left(25^{\circ} \mathrm{C}\right)$.

\subsection{Fabrication of PCL Microparticles via Electrospraying}

The experimental set-up of electrospraying (CAT000002, Electrospraying Instrument Kit Instruction Manual, Spraybase ${ }^{\circledR}$, AVECATS, Kildare, Ireland) used to produce PCL microparticles. During electrospraying, temperature and relative humidity were maintained at $25{ }^{\circ} \mathrm{C}$ and $45 \%$, respectively. Firstly, PCL solutions were added into an electrospraying pressure vessel, and a plastic tube was used to link a stainless steel nozzle (28 gauge, outer diameter: $360 \mu \mathrm{m}$, internal diameter: $180 \mu \mathrm{m})$ and pressure vessel. Then, the liquid was pumped from vessel to nozzle by air pressure. In this paper, the flow rate of PCL solutions was characterized by the dripping time for one single droplet from nozzle. The same dripping time for one single droplet from the nozzle indicates that the flow rate of PCL solutions is consistent. In most cases, for PCL/chloroform, PCL/glacial acetic acid, PCL/ethyl acetate and PCL/acetone, the dripping time for one single droplet was fixed at $5 \mathrm{~s}$; for PCL/anisole, the dripping time for one single droplet was fixed at $93 \mathrm{~s}$. Different dripping times for one single droplet can be obtained via adjusting the air pressure. The working distance from nozzle to collector was set to 9, 13, 17, 21 or $25 \mathrm{~cm}$. After setting dripping time and working distance, high voltage generated from a high-voltage generator was applied between the nozzle and collector ranging from 2 to $7 \mathrm{kV}$. The electrosprayed microparticles were collected onto glass slides for optical microscopy observation and aluminum foil for scanning electron microscopy analysis, with a collecting time of 3 and $10 \mathrm{~min}$, respectively.

\subsection{Measurements and Characterizations}

During electrospraying, the formation and shape of Taylor cone was observed and analysed via high-speed images captured by camera (Chameleon CMLN-13S2M-CS USB camera, FLIR Integrated Imaging Solutions $\mathrm{GmbH}$, Ludwigsburg, Germany).

The size and distribution of electrosprayed PCL microparticles were observed via optical microscopy (Motic BA410, Barcelona, Spain) linking with a Moticam 5 Digital Camera (Barcelona, Spain). The software Motic image plus 3.0 was used to capture the images, and the image analysis software (Image J, version 1.52j) was used to determine the mean diameter and sizes distribution. In order to obtain accurate results, 100-500 random PCL electrosprayed microparticles were selected to be measured and analyzed. The obtained data were statistically analyzed in a spreadsheet (Microsoft Excel 2011, Microsoft Company, Redmond, WA, USA) to calculate the mean diameter as well as the particle size distribution.

Scanning electron microscopy (SEM) (JEOL Model JSM-5900, Tokyo, Japan) was used to observe the surface morphology of eletrosprayed PCL microparticles. The accelerated voltage was $20 \mathrm{KV}$. The surfaces of PCL microparticles were coated with gold before analysis.

The surface tension $(\gamma, \mathrm{mN} / \mathrm{m})$ of PCL solutions were measured by Standard Wilhelmy's plate method based on a tensiometer from GBX Instruments (Romans sur Isere, France). The temperature and humidity of measurement were maintained at $25{ }^{\circ} \mathrm{C}$ and $45 \%$, respectively. The vessel was washed with detergent, placed in chromosulfuric acid overnight, washed with distilled water, and briefly flamed with a Bunsen burner prior to use. The platinum plate was rinsed in acetone and distilled water, and flamed before use. The accuracy of measurement was $\pm 0.1 \mathrm{mN} / \mathrm{m}$. All sample surface tension measurements were repeated 5 times per samples and each sample was prepared in triplicate. The average values and standard deviations were calculated. 
The electrical conductivities ( $K$, unit: $\mu \mathrm{S} / \mathrm{cm}$ ) of PCL electrospraying solutions were measured with a conductimeter type CDRV 62 (Tacussel electronique Instruments, Lyon, France). For each PCL solution, all sample surface tension measurements were repeated 5 times per sample and each sample was prepared in triplicate. The average values and standard deviations were calculated. The temperature and humidity of measurement were maintained at $25^{\circ} \mathrm{C}$ and $45 \%$, respectively.

The kinematic viscosity $\left(v\right.$, unit: $\left.\mathrm{mm}^{2} / \mathrm{s}\right)$ of PCL electrospraying solutions were measured by Ubbelohde viscometer (SI Analytics, Mainz, Germany). For each sample, the flow time $(t)$ in seconds was recorded, and kinematic viscosity was calculated from Equation (1):

$$
v=\alpha \times t
$$

where $t$ is the time in $\mathrm{s}$, and $\alpha$ the instrument constant related with the type of capillary. Two Ubbelohde viscometers with different capillaries were used according to the viscosity range of the solutions. For $0.84 \mathrm{~mm}$ capillary, $\alpha$ is 0.03 ; for $0.47 \mathrm{~mm}$ capillary, $\alpha$ is 0.003 . For each PCL solution, all sample surface tension measurements were repeated 5 times per sample and each sample was prepared in triplicate. The average values and standard deviations were calculated. The temperature of measurement was controlled at $25^{\circ} \mathrm{C}$ by a super thermostat.

\section{Results and Discussion}

\subsection{Physico-Chemical Properties of PCL Electrospraying Solutions}

In Table 1, the Hansen solubility parameters of different solvents as well as their solubility to PCL were given. The Hansen solubility parameters of all solvents used in our experiment are close to that of PCL $\left(\delta_{\mathrm{D}}, \delta_{\mathrm{H}}\right.$ and $\delta_{\mathrm{P}}$ of PCL is $17.0,8.3$ and 4.8 , respectively). Meanwhile, for most of them, their distances $\left(D_{\mathrm{s}}\right)$ to PCL in "Hansen space" were smaller than the radius of solubility sphere of PCL ( $R_{\mathrm{S}}$ ranges from 5.0 to 7.1 ) [35]. It indicates that all of them are suitable for dissolving PCL during electrospraying. For glacial acetic acid, anisole, and chloroform, the complete dissolution of PCL in these solvents can be held for a long time, even if at high PCL concentration. For ethyl acetate and acetone, at relatively high concentrations $(7 \mathrm{wt} \%$ and $10 \mathrm{wt} \%)$, there are some PCL solids precipitated from PCL solution after one week and four days, respectively. Therefore, glacial acetic acid, chloroform, and anisole are regarded as good solvents for dissolving PCL; and acetone and ethyl acetate are regarded as partial solvents for dissolving PCL.

During electrospraying, the formation and shape of Taylor cone, the break-up of charged droplets and the evaporation of solvent were governed by the physico-chemical properties of electrospraying (mainly including, surface tension, electrical conductivity, viscosity, and vapor pressure) and operating parameters. Table 1 also presents the effects of different solvents and different PCL concentrations on the physico-chemical properties of electrospraying solutions. In the cases of PCL/ethyl acetate, $\mathrm{PCL} /$ glacial acetic acid and PCL/acetone, the surface tension of solutions do not change significantly with the increasing of PCL concentration. For the couples of PCL/anisole and PCL/chloroform, the surface tension of solutions increases with the increasing of PCL concentration. Furthermore, $\mathrm{PCL} /$ anisole solutions have the higher surface tension compared with other solutions. The surface tensions of PCL/glacial acetic acid solutions are slightly higher than those of PCL/ethyl acetate, $\mathrm{PCL} /$ chloroform, and PCL/acetone solutions, which are close to each other.

Among five solvents, chloroform, anisole and ethyl acetate belong to low electrical conductivity solvents. Therefore, the electrical conductivities of PCL/chloroform, PCL/anisole, and PCL/ethyl acetate solutions are low (Table 1). In cases of PCL/glacial acetic acid and PCL/acetone solutions, their electrical conductivities are relatively higher because of the high electrical conductivity of the pure solvents. In addition, it decreases with the increasing of PCL concentration. With the increasing of PCL concentration, the kinematic viscosity of PCL solutions increases gradually for each solvent and also depends on the kind of solvent used for the solubilization. Thus, kinematic viscosity of the solutions 
follows the order: PCL/glacial acetic acid $>$ PCL/chloroform $>$ PCL/anisole $>$ PCL/ethyl acetate $>$ $\mathrm{PCL} /$ acetone.

Table 1. Hansen solubility parameters of different solvents and physico-chemical properties of electrospraying solutions obtained from different solvents as well as PCL concentrations.

\begin{tabular}{|c|c|c|c|c|c|c|}
\hline Property & PCL Concentration & Chloroform & Ethyl Acetate & Acetic Acid & Acetone & Anisole \\
\hline$\delta_{\mathrm{D}}{ }^{\mathrm{a}}$ & - & 18.2 & 15.8 & 14.5 & 15.7 & 17.8 \\
\hline$\delta_{\mathrm{H}}{ }^{\mathrm{a}}$ & - & 6.2 & 7.2 & 13.5 & 11.7 & 6.7 \\
\hline$\delta_{\mathrm{P}}{ }^{\mathrm{a}}$ & - & 6.3 & 5.3 & 8.0 & 5.3 & 4.1 \\
\hline$D_{\mathrm{S}}^{\mathrm{b}}$ & - & 1.9 & 4.0 & 8.4 & 5.7 & 2.4 \\
\hline PCL solubility & - & good & partial & good & partial & good \\
\hline Vapor pressure at $25^{\circ} \mathrm{C}(\mathrm{kPa})$ & - & 26.2 & 12.4 & 2.09 & 30.7 & 0.47 \\
\hline \multirow{5}{*}{ Surface tension $(\mathrm{mN} / \mathrm{m})$} & $1 \mathrm{wt} \%$ & $20.8 \pm 0.6$ & $22.8 \pm 0.5$ & $23.7 \pm 0.8$ & $21.6 \pm 0.3$ & $31.1 \pm 1.4$ \\
\hline & $3 \mathrm{wt} \%$ & $22.4 \pm 0.2$ & $21.8 \pm 0.4$ & $24.5 \pm 0.5$ & $21.0 \pm 0.6$ & $32.9 \pm 0.3$ \\
\hline & $5 \mathrm{wt} \%$ & $21.5 \pm 0.5$ & $22.4 \pm 0.6$ & $24.7 \pm 1.1$ & $21.5 \pm 0.3$ & $33.9 \pm 0.5$ \\
\hline & $7 \mathrm{wt} \%$ & $22.6 \pm 0.9$ & $22.1 \pm 0.6$ & $24.3 \pm 0.2$ & $20.9 \pm 0.3$ & $32.4 \pm 0.7$ \\
\hline & $10 \mathrm{wt} \%$ & $24.1 \pm 0.9$ & $21.5 \pm 0.5$ & $24.3 \pm 0.7$ & $20.2 \pm 1.0$ & $34.7 \pm 0.5$ \\
\hline \multirow{5}{*}{ Electrical conductivity $(\mu \mathrm{S} / \mathrm{cm})$} & $1 \mathrm{wt} \%$ & $<0.037$ & $<0.037$ & $0.251 \pm 0.032$ & $0.576 \pm 0.006$ & $<0.037$ \\
\hline & $3 \mathrm{wt} \%$ & $<0.039$ & $<0.038$ & $0.245 \pm 0.028$ & $0.481 \pm 0.058$ & $<0.039$ \\
\hline & $5 \mathrm{wt} \%$ & $<0.038$ & $<0.039$ & $0.235 \pm 0.026$ & $0.428 \pm 0.005$ & $<0.038$ \\
\hline & $7 \mathrm{wt} \%$ & $<0.040$ & $<0.037$ & $0.218 \pm 0.019$ & $0.323 \pm 0.001$ & $<0.040$ \\
\hline & $10 \mathrm{wt} \%$ & $<0.039$ & $<0.039$ & $0.202 \pm 0.016$ & $0.302 \pm 0.014$ & $<0.039$ \\
\hline \multirow{5}{*}{ Viscosity $\left(\mathrm{mm}^{2} / \mathrm{s}\right)$} & $1 \mathrm{wt} \%$ & $1.09 \pm 0.06$ & $0.73 \pm 0.05$ & $1.85 \pm 0.13$ & $0.53 \pm 0.03$ & $1.65 \pm 0.10$ \\
\hline & $3 \mathrm{wt} \%$ & $4.00 \pm 0.07$ & $1.56 \pm 0.01$ & $4.28 \pm 0.001$ & $0.95 \pm 0.05$ & $3.77 \pm 0.01$ \\
\hline & $5 \mathrm{wt} \%$ & $8.40 \pm 0.14$ & $3.21 \pm 0.01$ & $8.34 \pm 0.03$ & $2.06 \pm 0.12$ & $7.68 \pm 0.06$ \\
\hline & $7 \mathrm{wt} \%$ & $17.00 \pm 0.14$ & $6.13 \pm 0.01$ & $16.67 \pm 0.05$ & $3.47 \pm 0.01$ & $14.77 \pm 0.01$ \\
\hline & $10 \mathrm{wt} \%$ & $38.78 \pm 0.47$ & $16.61 \pm 0.02$ & $41.30 \pm 1.40$ & $11.15 \pm 0.05$ & $30.99 \pm 0.68$ \\
\hline
\end{tabular}

${ }^{a}: \delta_{D}, \delta_{H}$ and $\delta_{\mathrm{P}}$ are disperse part of Hansen solubility parameter, hydrogen part of Hansen solubility parameter and polar part of Hansen solubility parameter, respectively (unit: $\left.(\mathrm{MPa})^{1 / 2}\right) \cdot{ }^{\mathrm{b}}: D_{\mathrm{S}}$ is a distance between solvent $(\mathrm{S})$ and solute (PCL) in the "solubility space".

\subsection{The Cone-Jet Range of Applied Voltage}

Carrying out electrospraying under cone-jet mode is the prerequisite for preparing PCL electrosprayed particles with low mean diameter and narrow size distribution. The active control and design on the size, as well as the morphology of electrosprayed microparticles, may be achieved only under cone-jet mode. The main factor for determining the spraying mode during electrospraying is the applied voltage. With increasing it, the spraying mode changes from dripping mode to rapid dripping mode, then to unstable cone-jet mode, next to cone-jet mode, finally to multi-jet mode [41]. Therefore, a range of applied voltage exists for achieving cone-jet during electrospraying. In general, the cone-jet range of applied voltage is influenced by solution properties as well as operating parameters. In Table 2, the relationships among the cone-jet range of applied voltage, various solvents, different PCL concentration and different operating parameters are given. The electrical conductivity of the PCL solutions is the main parameters affecting the cone-jet ranges of the applied voltage. For low electrical conductive solutions, like PCL/chloroform, PCL/ethyl acetate, and PCL/anisole, their cone-jet ranges of the applied voltage are similar (approximately from 3.25 to $4.5 \mathrm{kV}$ ), while for $\mathrm{PCL} /$ glacial acetic acid and PCL/acetone, their cone-jet ranges of the applied voltage are higher than that of the other three solvents. It indicates that higher electrical conductivity of the solutions leads to a higher cone-jet range of applied voltage.

Furthermore, this range also correlates with the surface tension and the kinematic viscosity of the PCL solutions. Thus, for the solutions having the higher surface tension (PCL/glacial acetic acid), the cone-jet range of applied voltage is higher too. In Table 2, for PCL/chloroform, PCL/glacial acetic acid and PCL/anisole solutions, the lower limit as well as upper limit of the applied voltage increase with the increasing of PCL concentration, due to higher electrostatic forces required to overcome the viscoelastic one or high surface tension to guarantee the formation of Taylor cone. However, for PCL/ethyl acetate solutions, the upper limit of applied voltage decreases slightly with the increasing of PCL concentration. For high concentrations, cone-jet mode tends to change into asymmetrical and slanted with increasing the applied voltage. For PCL/acetone solutions, due to their too high electrical conductivities and too low viscosities, the lower limit and upper limit of the applied voltage decrease with the increasing of PCL concentration. The moderate viscosity is the 
prerequisite to maintain cone-jet. Due to too low viscosity, keeping cone-jet mode stable at low PCL concentration requires higher applied voltage. Meanwhile, the charge density in electrosprayed jet increases with the increase of applied voltage. At high PCL concentration, due to the thin charged jet and the accumulation of charges, the charge density in jet becomes too high, and then cone-jet changes into the multi-jet mode (unstable jet mode) under strong Coulomb repulsion.

Table 2. Cone-jet range of applied voltage of PCL electrosprayed solutions under different solvents, different PCL concentration and different operating parameters.

\begin{tabular}{|c|c|c|c|c|c|c|}
\hline \multicolumn{2}{|c|}{ Experimental Variables } & \multirow{2}{*}{$\begin{array}{c}\text { Chloroform (kV) } \\
3.25-4.25\end{array}$} & \multirow{2}{*}{$\begin{array}{c}\text { Ethyl Acetate (kV) } \\
3.25-4.75\end{array}$} & \multirow{2}{*}{$\begin{array}{c}\text { Glacial Acetic Acid (kV) } \\
4.00-5.75\end{array}$} & \multirow{2}{*}{$\begin{array}{c}\text { Acetone (kV) } \\
5.10-5.50\end{array}$} & \multirow{2}{*}{$\begin{array}{c}\text { Anisole (kV) } \\
3.30-4.25\end{array}$} \\
\hline \multirow{4}{*}{$\begin{array}{c}\text { PCL } \\
\text { concentration }^{a}\end{array}$} & $1 \mathrm{wt} \%$ & & & & & \\
\hline & $3 \mathrm{wt} \%$ & $3.32-4.25$ & $3.20-4.40$ & $4.20-5.90$ & $4.75-5.25$ & $3.40-4.50$ \\
\hline & $5 \mathrm{wt} \%$ & $3.50-4.40$ & $3.20-4.30$ & $4.25-6.15$ & $4.25-5.40$ & $3.40-4.25$ \\
\hline & $10 \mathrm{wt} \%$ & $3.70-4.50$ & $3.20-4.40$ & $4.25-6.40$ & $4.00-5.30$ & $3.50-4.80$ \\
\hline \multirow{3}{*}{$\begin{array}{l}\text { Working } \\
\text { distance }^{b}\end{array}$} & $9 \mathrm{~cm}$ & $3.50-4.20$ & $3.14-4.15$ & $3.87-5.25$ & $3.75-4.75$ & $3.30-4.10$ \\
\hline & $13 \mathrm{~cm}$ & $3.60-4.25$ & $3.17-4.25$ & $4.00-5.75$ & $4.00-5.00$ & $3.50-4.20$ \\
\hline & $25 \mathrm{~cm}$ & $3.70-4.40$ & $3.00-4.30$ & $4.40-6.40$ & $4.25-5.60$ & $3.50-4.50$ \\
\hline \multirow{4}{*}{$\begin{array}{l}\text { Dripping time } \\
\text { for one single } \\
\text { droplet }^{c}\end{array}$} & $9(104) \mathrm{s}$ & $3.60-4.40$ & $3.15-4.00$ & $4.00-6.00$ & $4.00-5.40$ & $3.50-4.25$ \\
\hline & $5(93) \mathrm{s}$ & $3.50-4.40$ & $3.20-4.50$ & $4.25-6.15$ & $4.25-5.40$ & $3.40-4.25$ \\
\hline & $4(67) \mathrm{s}$ & $3.80-4.60$ & $3.60-4.60$ & $4.25-6.30$ & $4.25-5.25$ & $3.50-4.25$ \\
\hline & $3(45) \mathrm{s}$ & $3.75-4.60$ & $3.60-4.60$ & $4.40-6.50$ & $4.25-5.00$ & $3.50-4.25$ \\
\hline
\end{tabular}

${ }^{\mathrm{a}}$ : Other experimental conditions were fixed as follows: working distance: $17 \mathrm{~cm}$, dripping time: $5 \mathrm{~s}$ (PCL/anisole: $93 \mathrm{~s}) ;$ b: Other experimental conditions were fixed as follows: PCL concentration: $5 \mathrm{wt} \%$, dripping time: $5 \mathrm{~s}$ (PCL/anisole: $93 \mathrm{~s}) ;{ }^{c}$ : The values in parentheses were the dripping time of PCL/anisole solution, other experimental conditions were fixed as follows: PCL concentration: $5 \mathrm{wt} \%$, working distance: $17 \mathrm{~cm}$.

Apart from solution properties, the cone-jet range of applied voltage also depends on the operating parameters. In Table 2, the lower and upper limits of cone-jet applied voltage increase with augmenting the working distance. Thus, it results in a decrease of the electric field strength, and needs to be adjusted to guarantee the formation of Taylor cone. The increasing trend of applied voltage in PCL/acetone and PCL/glacial acetic acid solutions are more significant compared with low electrical conductivity liquids, such as PCL/chloroform, PCL/ethyl acetate, and PCL/anisole. For high electrical conductivity liquids, they are more sensitive to the change in the strength of the electric field. The relationships between cone-jet applied voltage and flow rate of electrospraying liquid are also given in Table 2. Apart from PCL/acetone and PCL/anisole solutions, the lower limit and upper limit of applied voltage both increase with the accelerating of dripping time. It is due to the liquid gravity that will result in dripping mode being stronger under a higher flow rate of the liquid. Therefore, stronger applied voltage and electrostatic force are required to overcome stronger gravity and guarantee the formation of a Taylor cone. For PCL/acetone solutions, due to the accumulation of excessive charges and drastic coulomb repulsion, the cone-jet mode becomes unstable under higher flow rate. Therefore, their upper limits of applied voltage decrease gradually with the accelerating of dripping time.

\subsection{The Shape of Taylor Cone}

Under cone-jet mode, the shape of the Taylor cone affects the size as well as the distribution of electrosprayed microparticles. In this paper, the diameter of the charged jet and the distance between the nozzle and tip of the Taylor cone characterize the shape of the Taylor cone. The size as well as the size distribution of electrosprayed microparticles are smaller and more homogeneous when electrospraying is carried out under the thinner charged jet [42,43]. Meanwhile, the jet diameter also decreases with the decreasing of the distance between the nozzle and the tip of the Taylor cone [44,45]. Different shapes of the Taylor cone obtained from different solvents and different PCL concentrations are shown in Figure 1. For PCL/chloroform and PCL/ethyl acetate, the ideal Taylor cone with the small distance from nozzle to the tip of Taylor cone and thin jet diameter is easy to form under various PCL concentrations. For PCL/glacial acetic acid, the shape of Taylor cone is non-ideal at $1 \mathrm{wt} \%$ PCL 
(long distance between the nozzle and the tip of the Taylor cone and thick charged jet). It might be related to the relatively higher surface tension and insufficient viscosity. With the increasing of PCL concentration, the distance from the nozzle to the tip of the Taylor cone decreases gradually, and the shape of the Taylor cone becomes suitable for electrospraying. For PCL/acetone, at $1 \mathrm{wt} \% \mathrm{PCL}$, a rapid dripping mode with thick charged jet and long distance between the nozzle and the tip of Taylor cone is obtained due to too low viscosity of the solution. With the increasing of PCL concentration, the distance and the diameter of charged jet both decrease, and spray mode changes from rapid dripping mode to ideal cone-jet mode. For PCL/anisole, due to a too high surface tension and a low vapor pressure, their Taylor cone shapes are always non-ideal under various PCL concentrations. Furthermore, the diameter of charged jet in PCL/anisole decreases gradually with the increasing of PCL concentration.

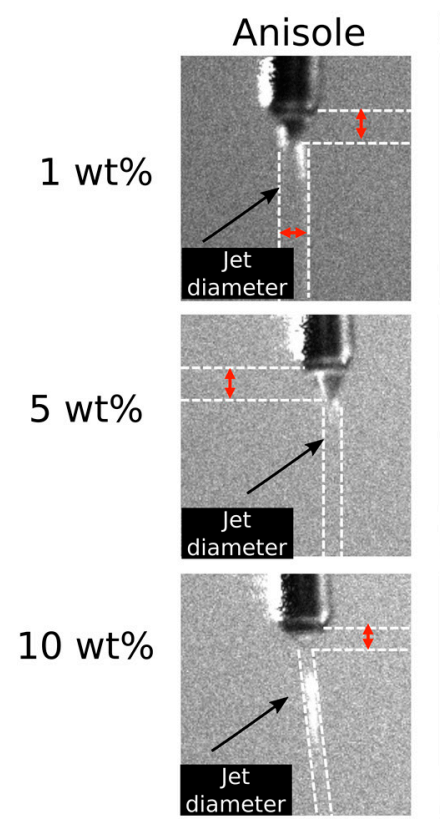

$3.75 \mathrm{kV}$
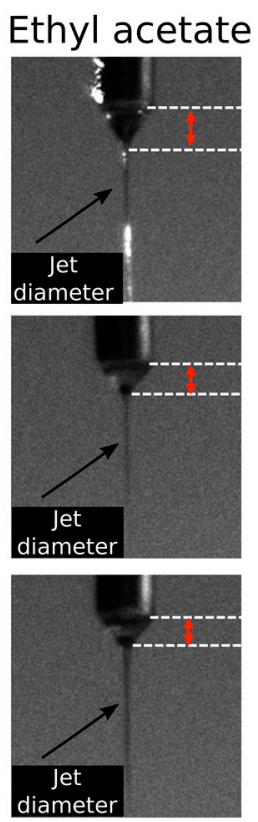

$3.75 \mathrm{kV}$
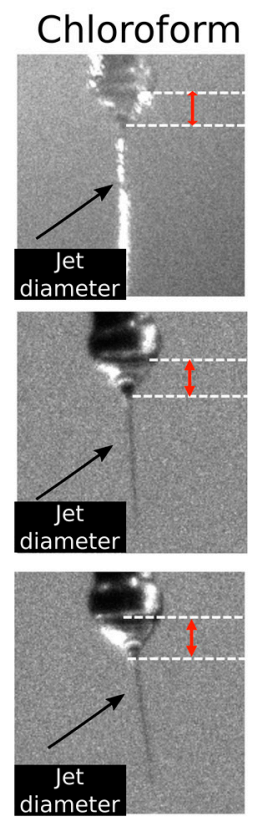

$3.75 \mathrm{kV}$
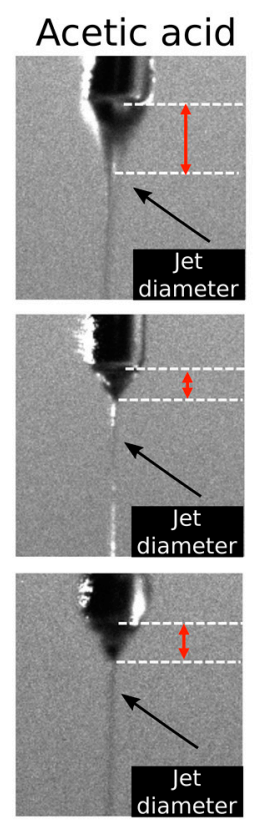

$5.25 \mathrm{kV}$
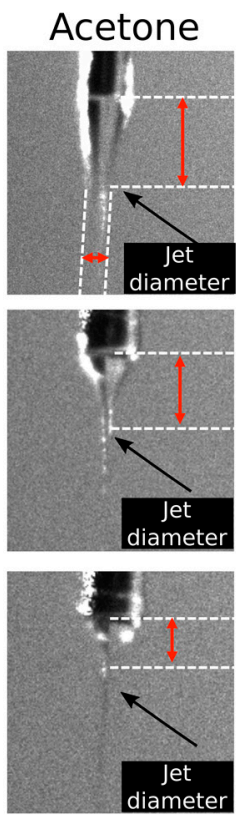

$4.75 \mathrm{kV}$

Figure 1. Images of Taylor cone obtained with anisole, ethyl acetate, chloroform, acetic acid and acetone at three PCL concentrations ( $1 \mathrm{wt} \%, 5 \mathrm{wt} \%$ and $10 \mathrm{wt} \%$ ) (working parameters: working distance $17 \mathrm{~cm}$, dripping time: $5 \mathrm{~s}$ (except for anisole: $93 \mathrm{~s})$ ).

Based on these images, medium viscosity and low surface tension values are prerequisites for the formation of Taylor cone with an adequate shape. With the increase of the viscosity, the distance from the nozzle to the tip of the Taylor cone and the jet diameter both decrease gradually, to promote the formation of a suitable cone-jet.

The shape of the Taylor cone also correlates with the operating parameters. In Figure 2, with the increase of working distance, the distance from the nozzle to the tip of the Taylor cone and jet diameter both increase gradually, particularly in PCL/acetone solutions, due to the reduction in the electric field and the electrostatic forces, and the shape as well as the stability are difficult to obtain. High viscosity is a main factor to keep the shape of Taylor cone stable; therefore, the changes in the shape of Taylor cone with the increasing of working distance among PCL/anisole, PCL/glacial acetic acid, PCL/ethyl acetate and PCL/chloroform solutions are lower than those of PCL/acetone solution ones.

The flow rate of electrosprayed liquid also influences the shape of the Taylor cone. In Figure 3, with the accelerating of dripping time for one single droplet, the distance from the nozzle to the tip of the Taylor cone as well as jet diameter both increase. It was closely related to the shortening of polarization time of droplets and the enhancing of droplet gravity during the formation of Taylor cone. Due to lower viscosity in PCL/acetone solutions, their Taylor cone stabilities are reduced. Therefore, 
the change in shape of the Taylor cone with the accelerating of dripping time is more significant for the $\mathrm{PCL} /$ acetone solution.

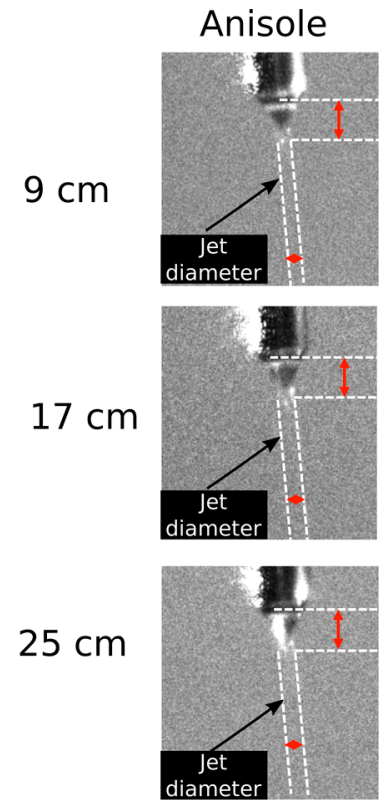

$3.75 \mathrm{kV}$
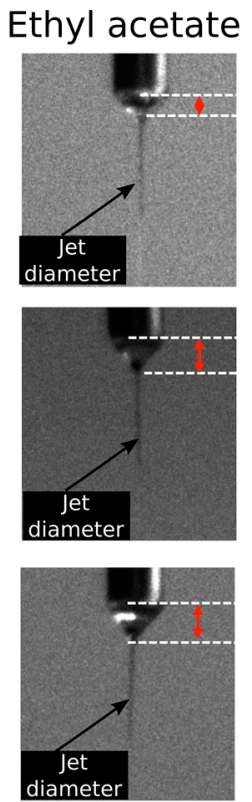

$3.75 \mathrm{kV}$
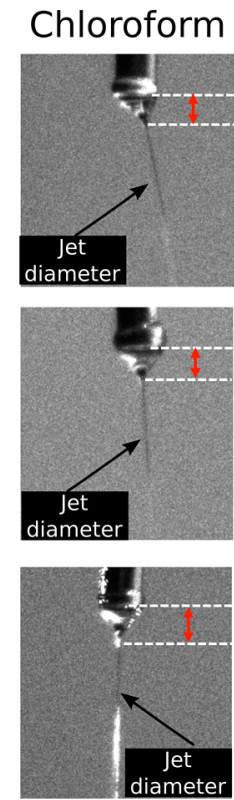

$3.75 \mathrm{kV}$
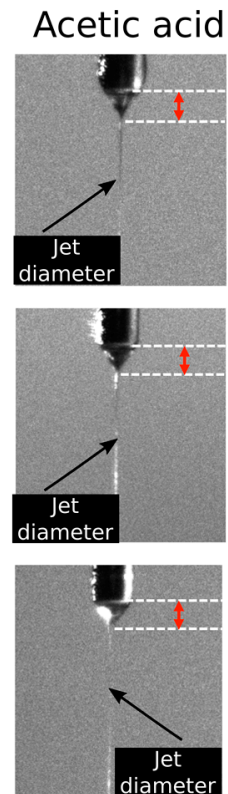

$5.25 \mathrm{kV}$
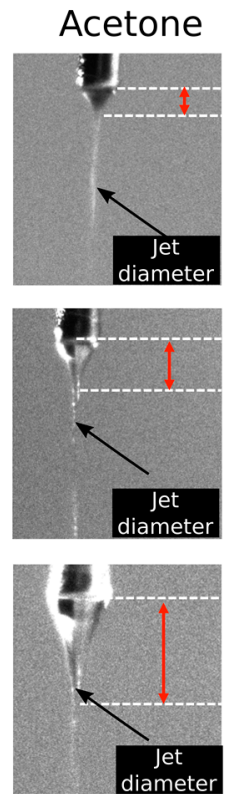

$4.75 \mathrm{kV}$

Figure 2. Images of Taylor cone obtained with anisole, ethyl acetate, chloroform, acetic acid and acetone at three working distances $(9,17$ and $23 \mathrm{~cm})$ at $5 \mathrm{wt} \%$ PCL concentrations for a dripping time of $5 \mathrm{~s}$ except for anisole (93s).

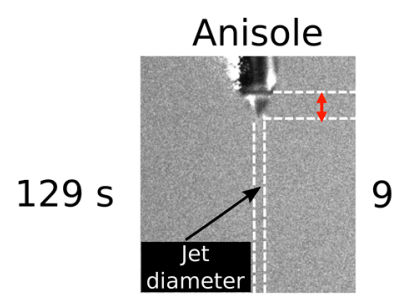

$93 \mathrm{~s}$

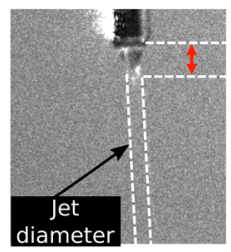

$45 \mathrm{~s}$

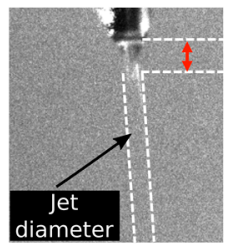

$3.75 \mathrm{kV}$
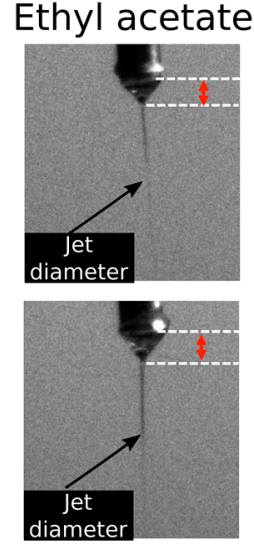

$3 \mathrm{~s}$

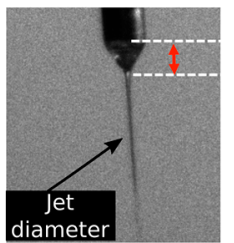

$3.75 \mathrm{kV}$
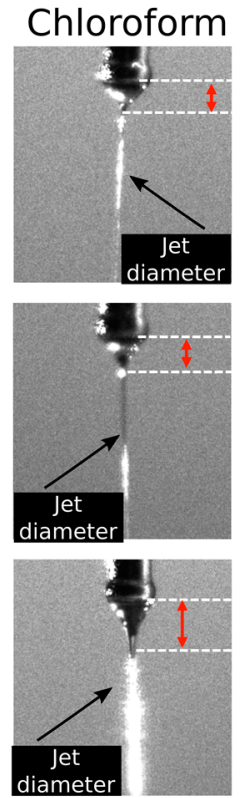

$3.75 \mathrm{kV}$
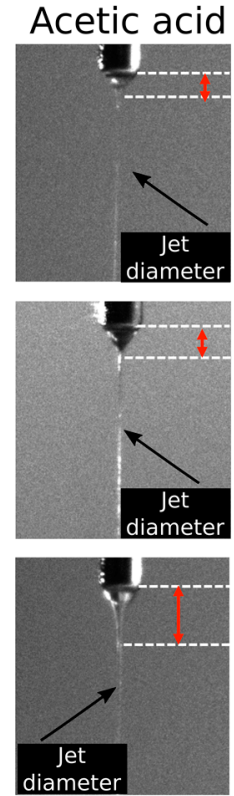

$5.25 \mathrm{kV}$
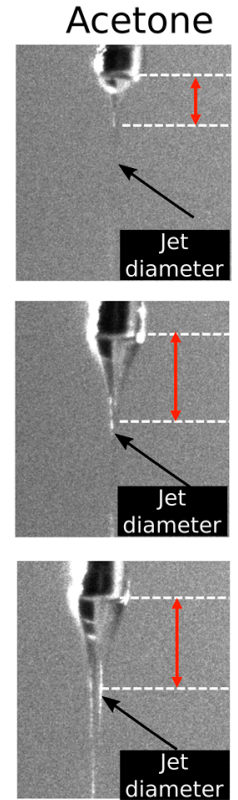

$4.75 \mathrm{kV}$

Figure 3. Images of Taylor cone obtained with anisole, ethyl acetate, chloroform, acetic acid and acetone at three dripping times $(129,93$, and $45 \mathrm{~s})$ at $5 \mathrm{wt} \%$ PCL concentrations and a fixed working distance of $17 \mathrm{~cm}$

\subsection{The Size and Distribution of PCL Microparticles}

Preparing micro- or nanoscale particles with narrow distribution is one of the main advantages of the electrospraying method. The size of electrosprayed microparticles mainly depends on solution properties and operating parameters. Furthermore, the shape of the Taylor cone influences the mean 
diameter and the particle size distribution of electrosprayed microparticles. Different mean diameters and different size distributions of PCL microparticles depend on the solvent choice as well as PCL concentration (Figure 4). For PCL/chloroform and PCL/ethyl acetate solutions, small (mean diameter: 7-8 $\mu \mathrm{m}$ ) and mono-dispersed distribution microparticles are obtained at $1 \mathrm{wt} \%$ PCL concentration, related to the moderate viscosities, ability of solvent evaporation, low surface tensions and electrical conductivities. Thus, for the solutions having low surface tension and moderate viscosity, adequate cone-jet is easy to form, and jet diameter is narrow. The break-up process of a thin charged jet is stable and uniform based on low electrical conductivity, when the solvents have a relatively high vapor pressure for complete evaporation, to lead to the formation of tiny microparticles. With the increasing of PCL concentration in these solvents, the mean diameter of microparticles increases gradually from 8 to $28 \mu \mathrm{m}$ and their size distributions broaden. It is related to the increasing of solid content in droplets and the enhancing entanglements among PCL chains under high PCL concentration. In this case, combined with their low electrical conductivities, the fine break-up of the charged jet will be suppressed. For PCL/glacial acetic acid and PCL/acetone solutions, the size distribution of PCL microparticles is poly-dispersed at low PCL concentration. Because of their higher electrical conductivities and thicker charged jet, the break-up process of the charged jet is drastic and unstable. The subsequent break-up of charged droplets also occurs during their flight to the collector. Therefore, the size distribution under this situation is inhomogeneous and indeed poly-dispersed. With the increasing of PCL concentration in glacial acetic acid, the mean diameter of small microparticles increases (from 4.58 to $7.63 \mu \mathrm{m}$ ), while that of large microparticles decreases (from 24.17 to $19.19 \mu \mathrm{m}$ ). Finally, bimodal-dispersed distribution turns into mono-dispersed distribution at $10 \mathrm{wt} \% \mathrm{PCL}$, and the mean diameter of PCL microparticles is about $15 \mu \mathrm{m}$. The situation in PCL/acetone solutions is similar to PCL/glacial acetic acid solutions, related to the stability of the break-up process of the charged jet. In addition, some PCL microparticles with too large size (diameter: $97 \mu \mathrm{m}$ ) were prepared from $3 \mathrm{wt} \% \mathrm{PCL} /$ acetone, due to unstable break-up of charged jet and quickness of the acetone evaporation. There is not enough time for PCL chains to condense together completely before the solidification of $\mathrm{PCL} /$ acetone droplets.

On the one hand, with the increasing of PCL concentration in glacial acetic acid or acetone, the electrical conductivities of solutions decrease, while their viscosities increase significantly. Then, the break-up process of the charged jet becomes stable and homogeneous. On the other hand, with the increasing of PCL concentration, the thickness of the charged jet obtained for solutions prepared from acetone or glacial acetic acid decreases, to promote the formation of electrosprayed particles with tiny size and mono-dispersed distribution. For PCL/anisole solutions, the mean diameter of microparticles is higher than those obtained from the other solvents. It is closely related with the diameter of charged jet and the slow evaporation of solvent. Due to too high surface tension and low vapor pressure, the diameter of the charged jet is large. Combined with low electrical conductivity, the fine break-up process of the charged jet is challenging to carry out. Meanwhile, due to residual solvent, microparticles tend to swell or aggregate together at the collector, which leads to large size and poly-dispersed distribution. The increase of the PCL concentration in the solutions allows the mean diameter to decrease significantly, which is related to the thinned charged jet and the reduction of solvent in droplets.

The mean diameter and size distribution of PCL electrosprayed microparticles prepared from different working distances with different solvents are presented in Figure 5. There are two opposite effects influencing on the mean diameter of electrosprayed particles introduced by the working distance [46]. The strength of the electric field between nozzle and collector weakens gradually with the increase of working distance. In this configuration, the formation of Taylor cone and the charged droplets break-up process are both suppressed, and then larger PCL microparticles are obtained under longer working distance. However, with the increasing of working distance, there will be longer time to carry out the complete evaporation of solvent during the flight of droplets to the collector. With the evaporation of solvent, PCL chains tend to condense and the size of electrosprayed 
microparticles decreases gradually [47]. In addition, due to the reduction of electric field, the break-up process also becomes more uniform and stable under longer working distance. Combined with the stimulated solvent evaporation, the size distributions of electrosprayed particles obtained from all solvents narrow gradually with the increasing of working distance, and mono-dispersed distributions are finally obtained.
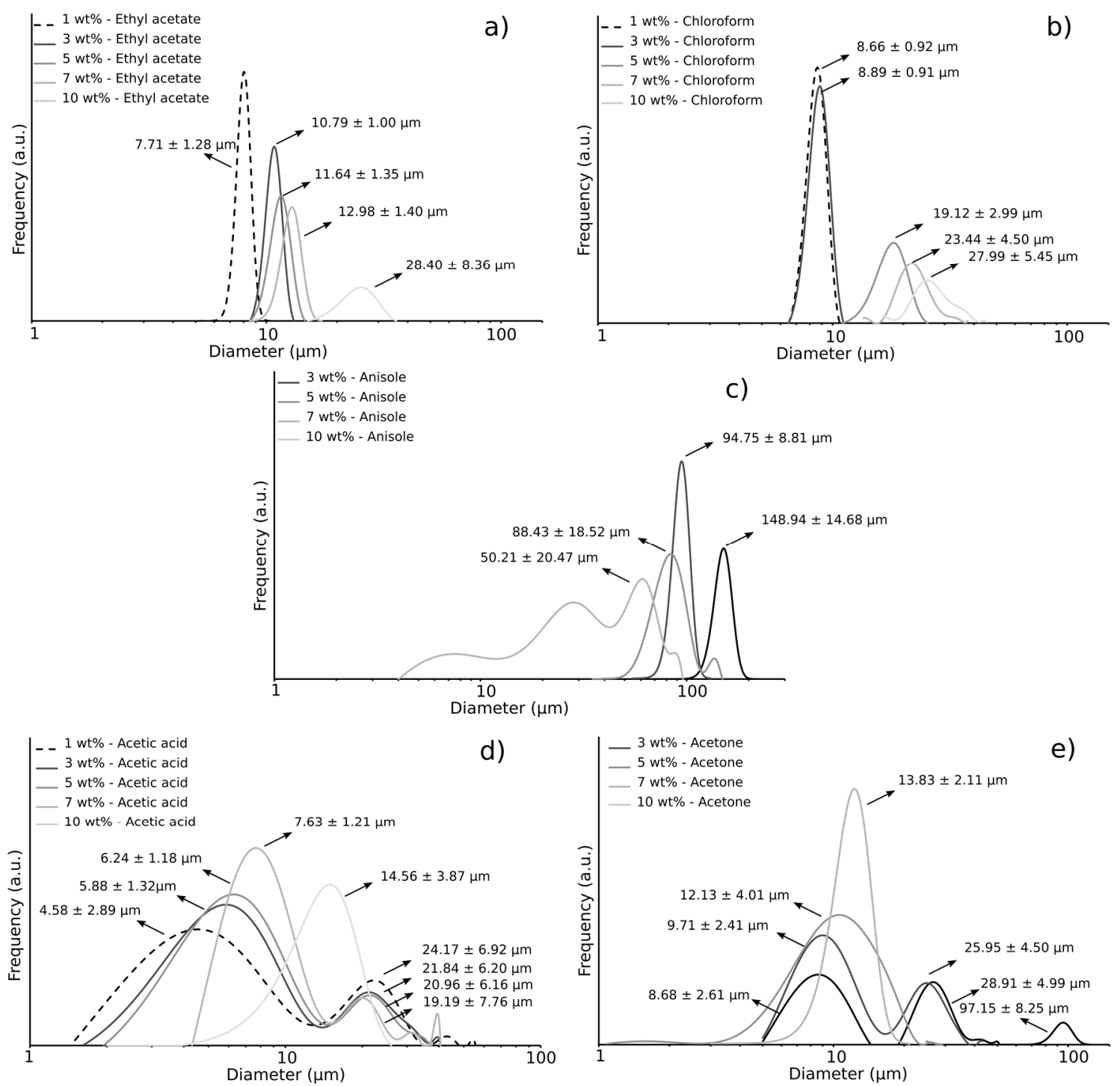

Figure 4. Mean diameter and size distribution of PCL electrosprayed microparticles obtained from ethyl acetate (a); chloroform (b); anisole (c); acetic acid (d); and acetone (e) for a working distance of $17 \mathrm{~cm}$, an applied voltage of $3.75 \mathrm{kV}$ (except for acetone: $4.75 \mathrm{kV}$, and glacial acetic acid: $5.25 \mathrm{kV}$, and a dripping time of $5 \mathrm{~s}$ (except for anisole, $93 \mathrm{~s}$ )).

For PCL/ethyl acetate solution, when working distance increases from 9 to $13 \mathrm{~cm}$, the mean diameter of PCL microparticles decreases from 19.08 to $11.64 \mu \mathrm{m}$. Thus, there is more solvent evaporating from droplets with longer flight distance. However, when working distance further increases, the mean diameter of microparticles does not change significantly. Thus, there is a trade-off between the two opposite effects on electrosprayed particles size introduced by the increasing of working distance. For PCL/chloroform solution, due to higher vapor pressure compared with $\mathrm{PCL} /$ ethyl acetate solution, the effect of solvent evaporation on particle size is stronger than that of the changing of electric field on particle size. Therefore, the mean diameter of microparticles decreases 
gradually with the increasing of working distance. For PCL/anisole solution, due to the low vapor pressure of anisole, the evaporation of solvent is difficult to carry out even under long working distance. Therefore, the effect of the changing of electric field on particle size is more important than that of solvent evaporation on particle size [48]. The mean diameter of electrosprayed particles increases gradually with the increasing of working distance.
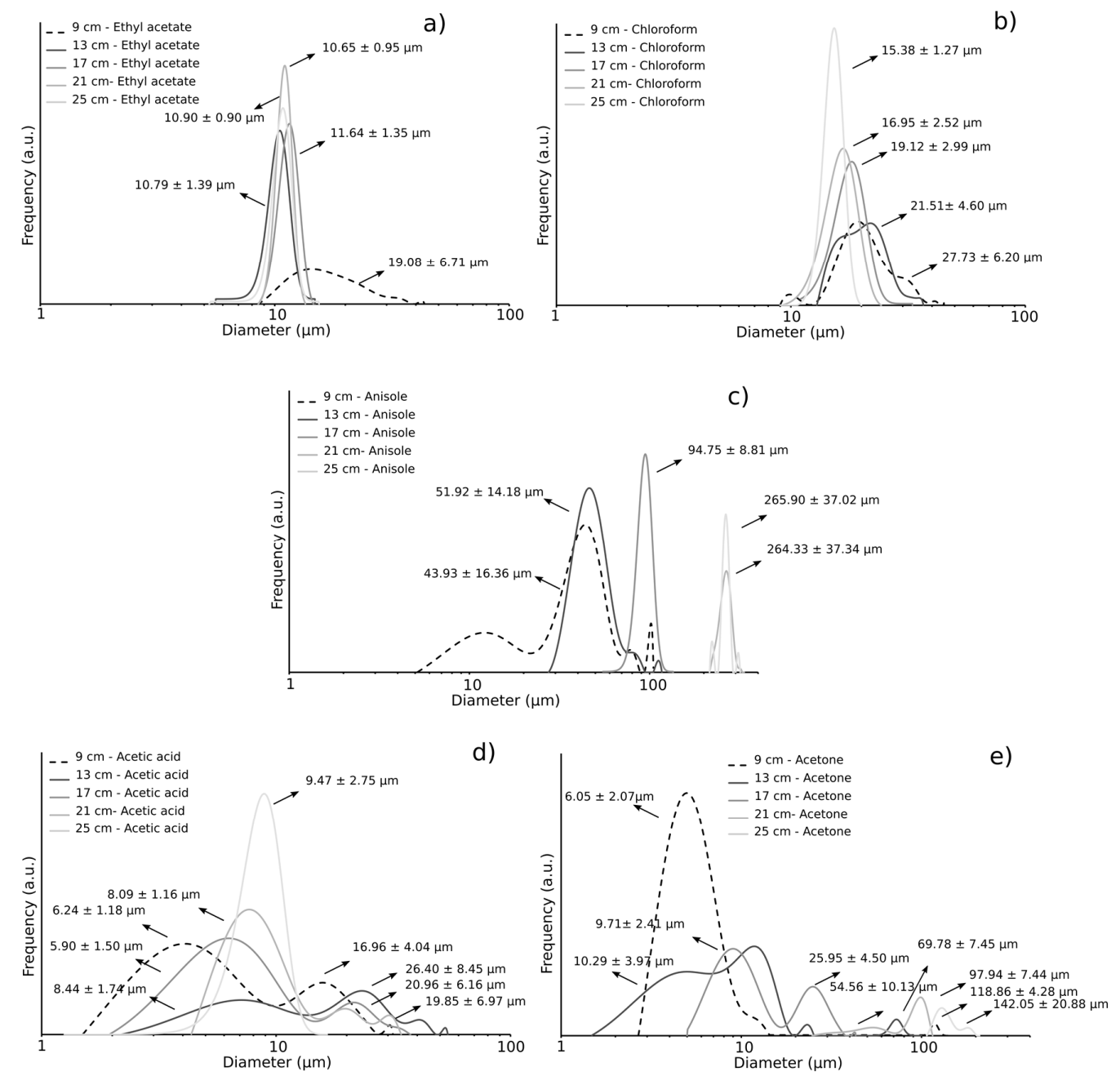

Figure 5. Mean diameter and size distribution of PCL electrosprayed microparticles obtained from ethyl acetate (a); chloroform (b); anisole (c); acetic acid (d); and acetone (e) for various working distances (9, 13, 17, 21 and $25 \mathrm{~cm}$, at $5 \mathrm{wt} \%$ PCL concentration for an applied voltage of $3.75 \mathrm{kV}$ (except for acetone: $4.75 \mathrm{kV}$, and glacial acetic acid: $5.25 \mathrm{kV}$ ), and a dripping time of $5 \mathrm{~s}$ (except for anisole, $93 \mathrm{~s}$ ).

For PCL/acetone solution, due to too stronger vapor pressure of acetone, the hardening of electrosprayed droplets during their flight to collector is very quick. Therefore, there is not enough time for the condensation of PCL chains during the solidification of electrosprayed particles. Under strong electric field at short working distance $(9 \mathrm{~cm})$, due to unstable break-up process of charged and quick hardening of electrosprayed droplets, tiny (mean diameter $\sim 6.05 \mu \mathrm{m}$ ) and large particles (mean diameter $118.86 \mu \mathrm{m}$ ) can be obtained. With the increasing of working distance, due to the reduction of electric field, the mean size of electrosprayed droplets increases gradually. Then, after quick solidification, the mean diameter of solid particles also increases with the increasing of working distance. For PCL/anisole and PCL acetone, the increasing of mean diameter of electrosprayed particles with the increasing of working distance also correlates with the increasing of charged jet diameter. For PCL/glacial acetic acid solution, the mean diameter of electrosprayed particles does not change significantly with the increasing of working distance. Thus, the effect of the changing of 
electric field on particle size is offset by the effect of solvent evaporation on particle size. In addition, based on the stable break-up process, sufficient solvent evaporation and condensation of PCL chains at $25 \mathrm{~cm}$, mono-dispersed and small microparticles can be prepared from glacial acetic acid.

The flow rate of the PCL solutions, related to the dripping time in this work, influences the granulometry of the obtained microparticles (Figure 6). For PCL/chloroform and PCL/ethyl acetate solutions with low electrical conductivity, the mean diameter, as well as the size distribution of PCL microparticles, is small and mono-dispersed under slow dripping time for one single droplet. With accelerating this dripping time, the mean diameter of PCL microparticles increases gradually, and the size distribution of PCL microparticles broadens [49], due to the obtention of the thicker charged jet under quicker dripping time. For PCL/acetone and PCL/glacial acetic acid solutions with high electrical conductivity, small and mono-dispersed PCL microparticles are obtained under slow dripping time due to thinner charged jet and a fine mist with the break-up of droplets. With accelerating dripping time, the diameter of charged jet increases gradually, and the break-up of charged droplets becomes unstable. Thus, the mean diameter of PCL microparticles increases and size distribution changes into bimodal-dispersed distribution. For PCL/anisole solutions, the mean diameter of PCL microparticles also increases with accelerating the dripping time.
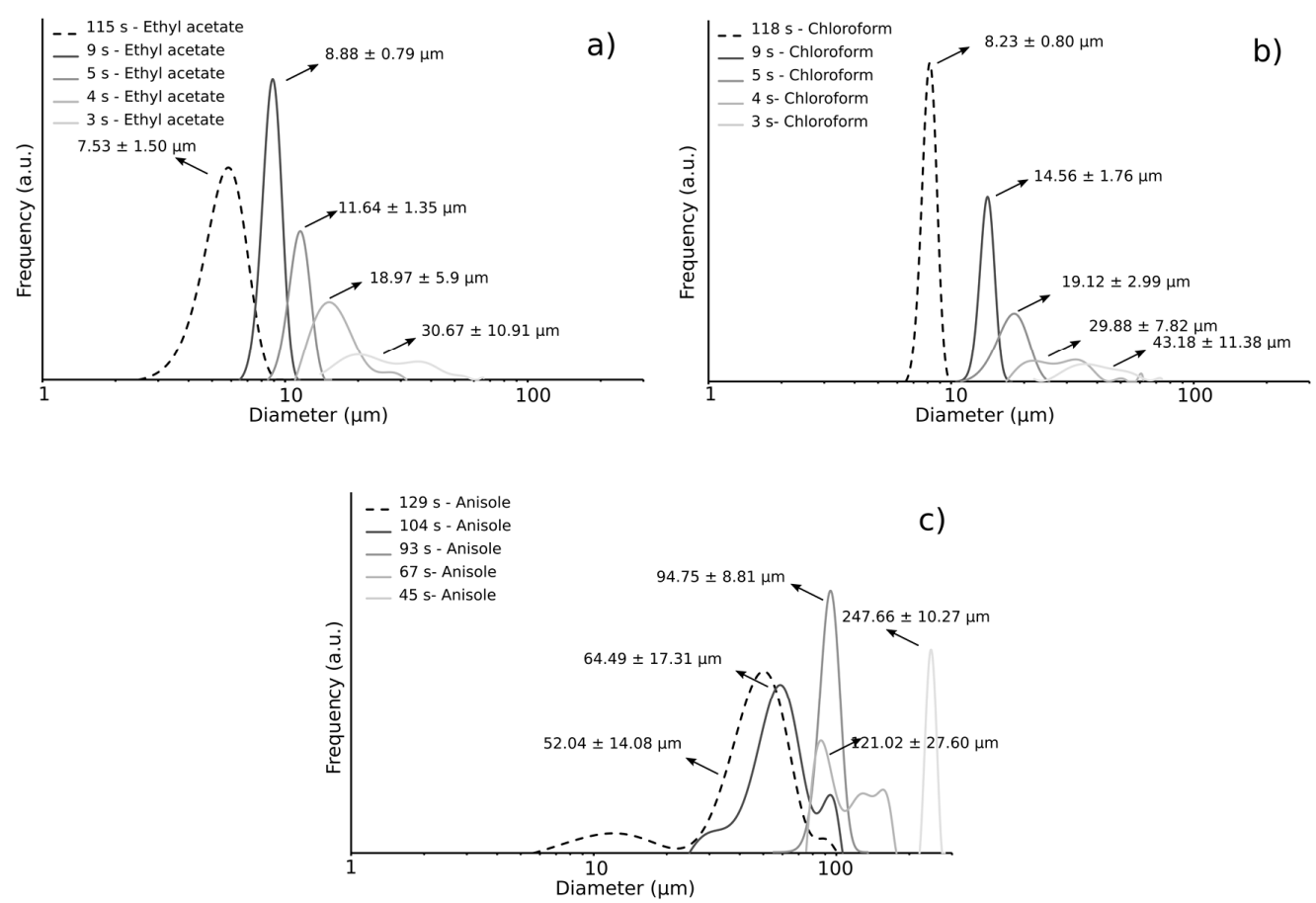

d)
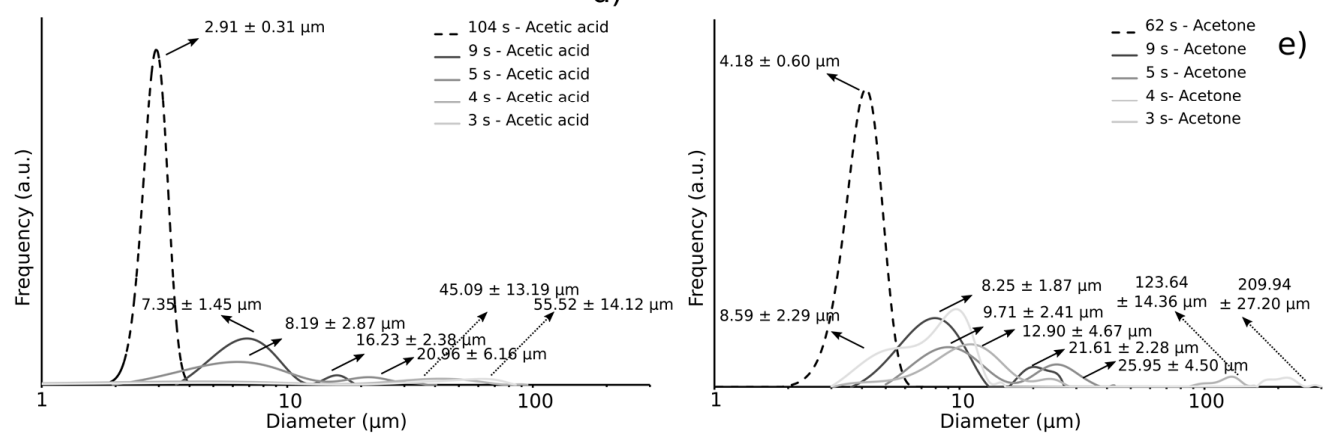

Figure 6. Mean diameter and size distribution of PCL electrosprayed microparticles obtained from ethyl acetate (a); chloroform (b); anisole (c); acetic acid (d); and acetone (e) for various dripping times, at $5 \mathrm{wt} \%$ PCL concentration for an applied voltage of $3.75 \mathrm{kV}$ (except for acetone: $4.75 \mathrm{kV}$, and glacial acetic acid: $5.25 \mathrm{kV}$ ), and a working distance of $17 \mathrm{~cm}$. 
Under cone-jet mode, the spray mode of electrospraying solutions changes from non-ideal cone-jet mode to ideal cone-jet mode with the increasing of the applied voltage [50]. Moreover, it influences the granulometry of the resulted microparticles (Figure 7). With the increasing of applied voltage, the mean diameter of PCL microparticles decreases and its distribution becomes narrow, due to the presence of stronger electrostatic forces and Coulomb repulsions among charged droplets, as observed in the cases of PCL/ethyl acetate and PCL/chloroform solutions. Therefore, mono-dispersed PCL microparticles with a mean diameter of about 11-15 $\mu \mathrm{m}$ are obtained at higher applied voltage. For PCL/anisole solution, with the increasing of applied voltage from 3.75 to $4.0 \mathrm{kV}$, there is an abnormal phenomenon occurring that the mean diameter of microparticles increases from 69.03 to $97.44 \mu \mathrm{m}$. It might correlate with the aggregation among PCL microparticles resulting from the residual solvent (anisole) in PCL microparticles. In the cases of PCL/glacial acetic acid and PCL/acetone solutions, the increase of the applied voltage allows for decreasing the particle size distribution, from poly-dispersed to bimodal or mono-dispersed distribution, in addition to the mean diameters.
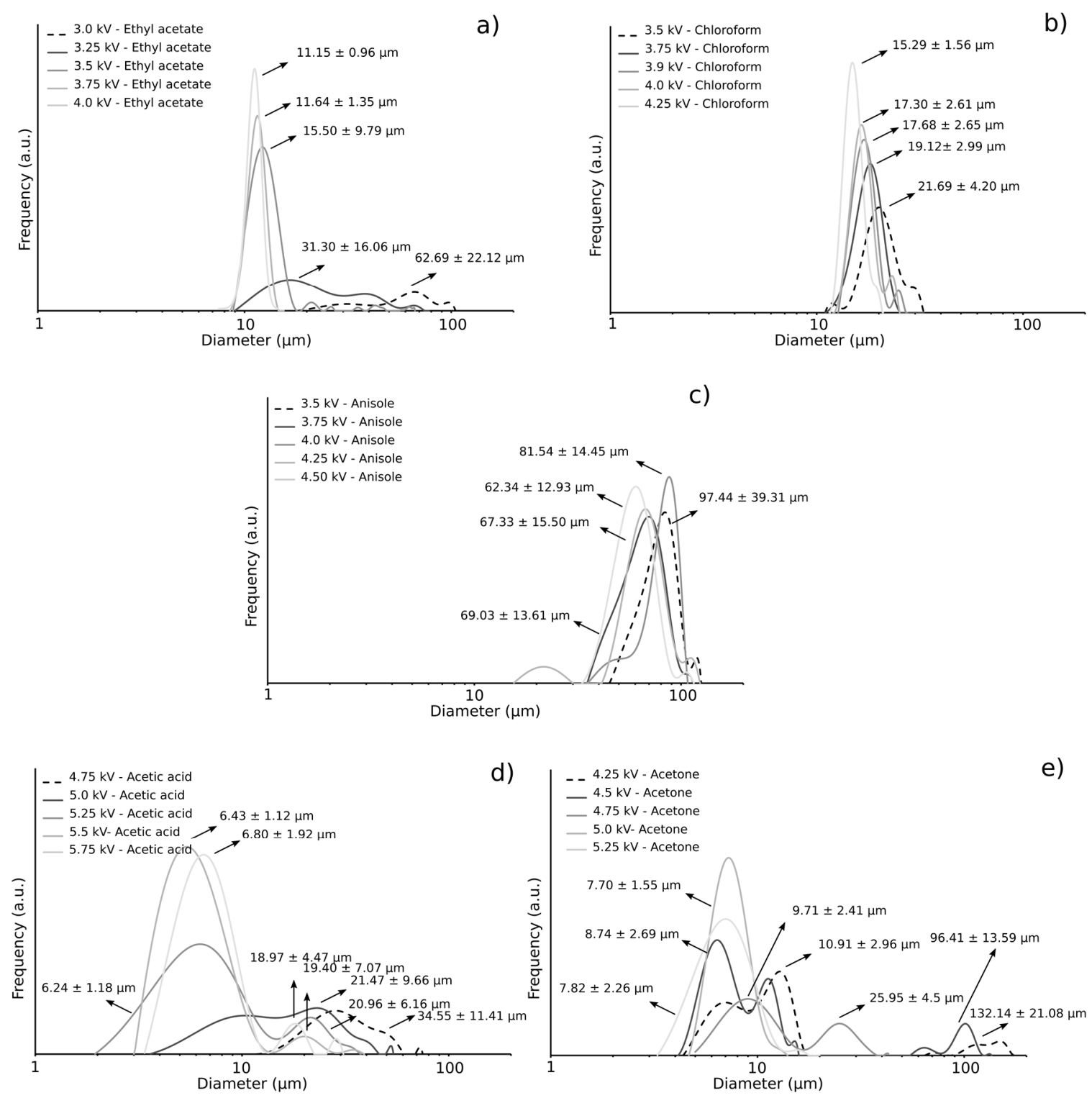

Figure 7. Mean diameter and size distribution of PCL electrosprayed microparticles obtained from ethyl acetate (a); chloroform (b); anisole (c); acetic acid (d); and acetone (e) for a dripping time of $5 \mathrm{~s}$ (except for anisole, $93 \mathrm{~s}$ ), at various applied voltages, and a working distance of $17 \mathrm{~cm}$. 


\subsection{The Morphology of PCL Microparticles}

The morphology of electrosprayed PCL microparticles mainly depends on the phase separation between solvent and PCL as well as the solvent evaporation [51-54], and therefore is affected by the solvent choice and the operating parameters. In general, for low vapor pressure solvents, their evaporations during electrospraying are slow. Then, there is enough time for the condensation of polymer chains and phase separation between solvent and PCL matrix before the solidification of electrosprayed droplets. Therefore, nonporous and smooth microparticles (Figure 8(d1-d3)) can be obtained from glacial acetic acid $\left(2.09 \mathrm{kPa}\right.$ at $\left.25^{\circ} \mathrm{C}\right)$. However, some residual solvent in microparticles after reaching the collector is also caused by the slowness of solvent evaporation. Due to the residual solvent, microparticles tend to swell and further aggregate together to form blocks or films. Under short working distance, quick dripping time and high PCL concentration, due to further depression of solvent evaporation, this phenomenon is more obvious (Figure $8(\mathrm{~d} 2, \mathrm{~d} 3))$. For PCL/anisole $(0.47 \mathrm{kPa}$ at $25{ }^{\circ} \mathrm{C}$ ), due to lower vapor pressure, the evaporation rate of solvent during electrospraying is slower. Therefore, there is more solvent remaining in collected microparticles. After the swelling and aggregation among them, only PCL films can be obtained (Figure 8e).

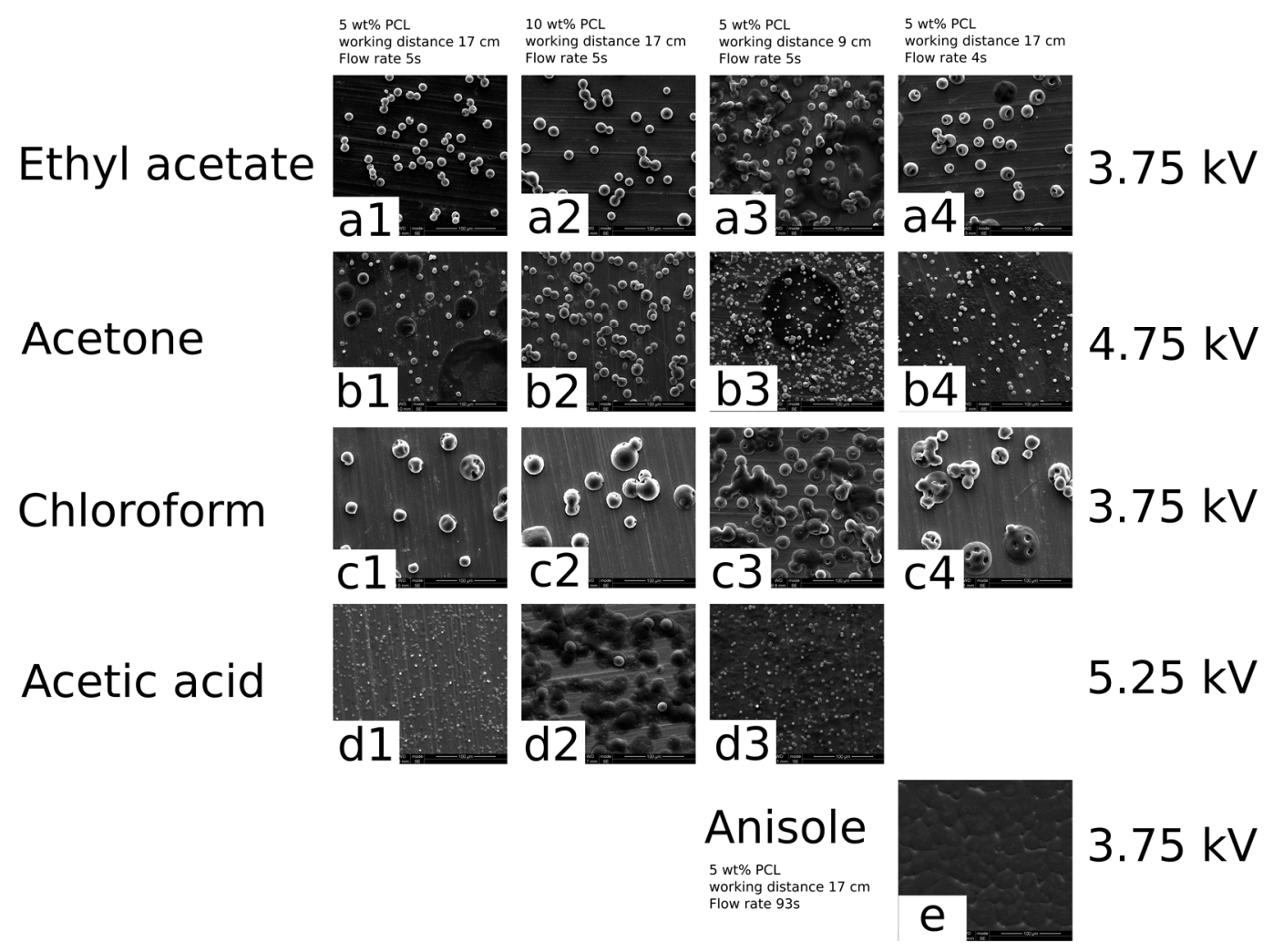

Figure 8. SEM images of PCL microparticles obtained from different solvent and different operating parameters (the caption in each figure presents the experimental conditions. For example, ethyl acetate- $5 \mathrm{wt} \%-17 \mathrm{~cm}-5 \mathrm{~s}$ presents that the solvent system is ethyl acetate; PCL concentration is $5 \mathrm{wt} \%$; working distance is $17 \mathrm{~cm}$; dripping time for one droplet is $5 \mathrm{~s}$. For figures (a1-a4, c1-c4 and e), the applied voltage is $3.75 \mathrm{kV}$. For figure (b1-b4), the applied voltage is $4.75 \mathrm{kV}$. For figure (d1-d3), the applied voltage is $5.25 \mathrm{kV}$. Scale bar: $100 \mu \mathrm{m}$ ).

For high vapor pressure solvents, their evaporations during electrospraying are quick. Before complete phase separation between solvent and PCL as well as sufficient condensation among PCL chains, solvent had volatilized quickly from electrosprayed droplets and left some pores, gaps and collapses on solid particles. Therefore, porous PCL microparticles can be prepared from chloroform $\left(26.2 \mathrm{kPa}\right.$ at $\left.25^{\circ} \mathrm{C}\right)$ and ethyl acetate $\left(12.4 \mathrm{kPa}\right.$ at $\left.25^{\circ} \mathrm{C}\right)$. Due to the differences between their vapour 
pressures, cherry-like PCL microparticles with a single pore are observed from a $5 \mathrm{wt} \% \mathrm{PCL} / \mathrm{ethyl}$ acetate solution (Figure 8(a1)). At the same concentration range in chloroform, wrinkle and porous structures are obtained (Figure 8(c1)). With the increasing of PCL concentration, due to the reduction of vapor pressure and the increasing of solid content in electrosprayed droplets, porous morphology tends to convert into nonporous morphology (Figure $8(\mathrm{a} 2, \mathrm{c} 2)$ ). With the decreasing of working distance, the evaporation of chloroform or ethyl acetate becomes incomplete. Therefore, PCL microparticles tend to aggregate together due to residual solvent in microparticles, and some PCL blocks or films with irregular shape and pores can be prepared eventually (Figure 8(a3,c3)). Meanwhile, with the accelerating of dripping time, the porous structure on PCL microparticles obtained from ethyl acetate or chloroform becomes more obvious (Figure $8(\mathrm{a} 4, \mathrm{c} 4)$ ).

For PCL/acetone solution, although it has higher vapor pressure $\left(30.7 \mathrm{kPa}\right.$ at $\left.25^{\circ} \mathrm{C}\right)$, nonporous and smooth particle surface can be fabricated (Figure 8(b1)). Thus, partial solubility of PCL in acetone leads to complete phase separation before the evaporation of acetone. Meanwhile, combining complete phase separation with quicker solvent evaporation, the morphology of PCL microparticles prepared from acetone keeps unchanged with the evolution of PCL concentration, working distance or dripping time. In addition, due to too high vapor pressure, some microparticles with irregular shape can be fabricated from acetone.

\section{Conclusions}

The effects of solution properties and operating parameters on the formation of electrosprayed PCL microparticles has been clarified. The applied voltage range for cone-jet mainly depends on the electrical conductivity of electrospraying solution. Solution with higher electrical conductivity needs higher applied voltage to achieve cone-jet during electrospraying. Meanwhile, ideal cone-jet mode is easier to obtain under high viscosity and low surface tension. With the increasing of viscosity, the stability of ideal Taylor cone also improved gradually. Furthermore, the shape of Taylor cone also influences the size and distribution of PCL electrosprayed microparticles. In general, under a short distance from nozzle to the tip of Taylor cone and thin charged jet, small and homogeneous microparticles can be prepared.

For electrospraying liquids with mild electrical conductivity, mono-dispersed microparticles can be fabricated. Their sizes closely correlate to their surface tensions and viscosities. For chloroform and ethyl acetate, small mean diameter of about several microns can be obtained from $1 \mathrm{wt} \%$ PCL concentration, and particle size increases with the increasing of PCL concentration. For anisole, large mean diameter of about hundreds of microns can be obtained from $1 \mathrm{wt} \%$ PCL concentration, and particle size decreases with the increasing of PCL concentration. For electrospraying liquid with high electrical conductivity, microparticles with poly-dispersed distribution can be obtained at low PCL concentration. With the increasing of PCL concentration, size distribution of microparticles narrows gradually. At $10 \mathrm{wt} \% \mathrm{PCL}$, mono-dispersed microparticles with about $15 \mu \mathrm{m}$ mean diameter can be prepared from glacial acetic acid or acetone. Furthermore, for all electrospraying liquids, mono-dispersed microparticles are easier to fabricate under long working distance. During the solidification of electrosprayed droplets, insufficient condensation of polymer chains or remaining solvent in collected particles both introduce large mean diameter. Only with the sufficient condensation of polymer chains and the complete evaporation of solvent carried out simultaneously can tiny electrosprayed particles be prepared. In addition, the mean diameter of all PCL microparticles decreases with the increasing of applied voltage or dripping time. Meanwhile, their size distributions also narrow gradually. For the morphology of electrosprayed PCL microparticles, the slowness of solvent evaporation or complete phase separation between solvent and PCL is good for obtaining nonporous and smooth morphology. When the evaporation rate of solvent is faster than the rate of phase separation, particles with porous and wrinkle morphology can be obtained. Finally, among four green solvents, ethyl acetate is a better substitution of chloroform for preparing PCL microparticles via electrospraying. 
Author Contributions: Conceptualization, S.Z., F.S. and C.C.; Formal Analysis, S.Z., C.C., and F.S.; Writing-Original Draft Preparation, S.Z. and F.S.; Writing—Review and Editing, S.Z., C.C. and F.S.

Funding: This research received no external funding.

Acknowledgments: We are very grateful to Sichuan University (Chengdu, China) for microscopy analyses (SEM) and the technical and financial support from GEMTEX laboratory (Lille, France). The author Shengchang Zhang is also thankful for the financial support for a PhD from the China Scholarship Council (CSC).

Conflicts of Interest: The authors declare no conflict of interest.

\section{References}

1. Bock, N.; Dargaville, T.R.; Woodruff, M.A. Electrospraying of polymers with therapeutic molecules: State of the art. Prog. Polym. Sci. 2012, 37, 1510-1551. [CrossRef]

2. Huang, L.-Y.; Yu, D.-G.; Branford-White, C.; Zhu, L.-M. Sustained release of ethyl cellulose micro-particulate drug delivery systems prepared using electrospraying. J. Mater. Sci. 2011, 47, 1372-1377. [CrossRef]

3. Fukui, Y.; Maruyama, T.; Iwamatsu, Y.; Fujii, A.; Tanaka, T.; Ohmukai, Y.; Matsuyama, H. Preparation of monodispersed polyelectrolyte microcapsules with high encapsulation efficiency by an electrospray technique. Colloids Surf. Physicochem. Eng. Asp. 2010, 370, 28-34. [CrossRef]

4. Jaworek, A.; Sobczyk, A.T.; Krupa, A. Electrospray application to powder production and surface coating. J. Aerosol Sci. 2018, 125, 57-92. [CrossRef]

5. Wang, H.; Li, W.; Li, Z. A facile strategy for preparing pcl/peg block copolymer microspheres via electrospraying as coatings for cotton fabrics. Macromol. Mater. Eng. 2018, 303, 1800164. [CrossRef]

6. Jaworek, A. Micro- and nanoparticle production by electrospraying. Powder Technol. 2007, 176, 18-35. [CrossRef]

7. Bock, N.; Woodruff, M.A.; Hutmacher, D.W.; Dargaville, T.R. Electrospraying, a reproducible method for production of polymeric microspheres for biomedical applications. Polymers 2011, 3, 131-149. [CrossRef]

8. Nath, S.D.; Son, S.; Sadiasa, A.; Min, Y.K.; Lee, B.T. Preparation and characterization of plga microspheres by the electrospraying method for delivering simvastatin for bone regeneration. Int. J. Pharm. 2013, 443, 87-94. [CrossRef]

9. Zhang, Q.; Liu, J.; Wang, X.; Li, M.; Yang, J. Controlling internal nanostructures of porous microspheres prepared via electrospraying. Colloid Polym. Sci. 2010, 288, 1385-1391. [CrossRef]

10. Massella, D.; Celasco, E.; Salaün, F.; Ferri, A.; Barresi, A. Overcoming the limits of flash nanoprecipitation: Effective loading of hydrophilic drug into polymeric nanoparticles with controlled structure. Polymers 2018, 10, 1092. [CrossRef]

11. Enayati, M.; Ahmad, Z.; Stride, E.; Edirisinghe, M. Size mapping of electric field-assisted production of polycaprolactone particles. J. R. Soc. Interface 2010, 7, S393-S402. [CrossRef] [PubMed]

12. Smeets, A.; Clasen, C.; Van den Mooter, G. Electrospraying of polymer solutions: Study of formulation and process parameters. Eur. J. Pharm. Biopharm. 2017, 119, 114-124. [CrossRef] [PubMed]

13. Xie, J.; Wang, C.H. Encapsulation of proteins in biodegradable polymeric microparticles using electrospray in the taylor cone-jet mode. Biotechnol. Bioeng. 2007, 97, 1278-1290. [CrossRef]

14. Enayati, M.; Chang, M.-W.; Bragman, F.; Edirisinghe, M.; Stride, E. Electrohydrodynamic preparation of particles, capsules and bubbles for biomedical engineering applications. Colloids Surf. Physicochem. Eng. Asp. 2011, 382, 154-164. [CrossRef]

15. Hartman, R.P.A.; Brunner, D.J.; Camelot, D.M.A.; Marijnissen, J.C.M.; Scarlett, B. Electrohydrodynamic atomization in the cone-jet mode physical modeling of the liquid cone and jet. J. Aerosol Sci. 1999, 30, 823-849. [CrossRef]

16. Jaworek, A. Electrostatic micro- and nanoencapsulation and electroemulsification: A brief review. J. Microencapsul. 2008, 25, 443-468. [CrossRef] [PubMed]

17. Zhang, S.; Kawakami, K. One-step preparation of chitosan solid nanoparticles by electrospray deposition. Int. J. Pharm. 2010, 397, 211-217. [CrossRef] [PubMed]

18. Cloupeau, M.; Prunet-Foch, B. Electrohydrodynamic spraying functioning modes: A critical review. J. Aerosol Sci. 1994, 25, 1021-1036. [CrossRef]

19. Xu, Y.; Skotak, M.; Hanna, M. Electrospray encapsulation of water-soluble protein with polylactide. I. Effects of formulations and process on morphology and particle size. J. Microencapsul. 2006, 23, 69-78. [CrossRef] 
20. Stachewicz, U.; Yurteri, C.U.; Frits Dijksman, J.; Marijnissen, J.C.M. Single event electrospraying of water. J. Aerosol Sci. 2010, 41, 963-973. [CrossRef]

21. De La Mora, J.F.; Loscertales, I.G. The current emitted by highly conducting taylor cones. J. Fluid Mech. 2006, 260, 155-184. [CrossRef]

22. Rosell-Llompart, J.; Fernández de la Mora, J. Generation of monodisperse droplets 0.3 to $4 \mu \mathrm{m}$ in diameter from electrified cone-jets of highly conducting and viscous liquids. J. Aerosol Sci. 1994, 25, 1093-1119. [CrossRef]

23. Chen, D.-R.; Pui, D.Y.H.; Kaufman, S.L. Electrospraying of conducting liquids for monodisperse aerosol generation in the $4 \mathrm{~nm}$ to $1.8 \mu \mathrm{m}$ diameter range. J. Aerosol Sci. 1995, 26, 963-977. [CrossRef]

24. Sinha, V.R.; Bansal, K.; Kaushik, R.; Kumria, R.; Trehan, A. Poly- $\epsilon$-caprolactone microspheres and nanospheres: An overview. Int. J. Pharm. 2004, 278, 1-23. [CrossRef] [PubMed]

25. Woodruff, M.A.; Hutmacher, D.W. The return of a forgotten polymer-Polycaprolactone in the 21st century. Prog. Polym. Sci. 2010, 35, 1217-1256. [CrossRef]

26. Dash, T.K.; Konkimalla, V.B. Poly-small je, ukrainian-caprolactone based formulations for drug delivery and tissue engineering: A review. J. Control. Release 2012, 158, 15-33. [CrossRef] [PubMed]

27. Lasprilla-Botero, J.; Torres-Giner, S.; Pardo-Figuerez, M.; Álvarez-Láinez, M.; Lagaron, J. Superhydrophobic bilayer coating based on annealed electrospun ultrathin poly( $\varepsilon$-caprolactone) fibers and electrosprayed nanostructured silica microparticles for easy emptying packaging applications. Coatings 2018, 8, 173. [CrossRef]

28. Klose, D.; Siepmann, F.; Elkharraz, K.; Krenzlin, S.; Siepmann, J. How porosity and size affect the drug release mechanisms from plga-based microparticles. Int. J. Pharm. 2006, 314, 198-206. [CrossRef]

29. Dunne, M.; Corrigan, O.I.; Ramtoola, Z. Influence of particle size and dissolution conditions on the degradation properties of polylactide-co-glycolide particles. Biomaterials 2000, 21, 1659-1668. [CrossRef]

30. Gaumet, M.; Vargas, A.; Gurny, R.; Delie, F. Nanoparticles for drug delivery: The need for precision in reporting particle size parameters. Eur. J. Pharm. Biopharm. 2008, 69, 1-9. [CrossRef]

31. Lee, Y.H.; Mei, F.; Bai, M.Y.; Zhao, S.; Chen, D.R. Release profile characteristics of biodegradable-polymer-coated drug particles fabricated by dual-capillary electrospray. J. Control. Release 2010, 145, 58-65. [CrossRef] [PubMed]

32. Gao, Y.; Bai, Y.; Zhao, D.; Chang, M.-W.; Ahmad, Z.; Li, J.-S. Tuning microparticle porosity during single needle electrospraying synthesis via a non-solvent-based physicochemical approach. Polymers 2015, 7, 2701-2710. [CrossRef]

33. Gao, Y.; Zhao, D.; Chang, M.-W.; Ahmad, Z.; Li, J.-S. Optimising the shell thickness-to-radius ratio for the fabrication of oil-encapsulated polymeric microspheres. Chem. Eng. J. 2016, 284, 963-971. [CrossRef]

34. Wu, Y.; Kennedy, S.J.; Clark, R.L. Polymeric particle formation through electrospraying at low atmospheric pressure. J. Biomed. Mater. Res. B Appl. Biomater. 2009, 90, 381-387. [CrossRef] [PubMed]

35. Bordes, C.; Freville, V.; Ruffin, E.; Marote, P.; Gauvrit, J.Y.; Briancon, S.; Lanteri, P. Determination of poly(epsilon-caprolactone) solubility parameters: Application to solvent substitution in a microencapsulation process. Int. J. Pharm. 2010, 383, 236-243. [CrossRef] [PubMed]

36. Bodnár, E.; Grifoll, J.; Rosell-Llompart, J. Polymer solution electrospraying: A tool for engineering particles and films with controlled morphology. J. Aerosol Sci. 2018, 125, 93-118. [CrossRef]

37. Pawar, A.; Thakkar, S.; Misra, M. A bird's eye view of nanoparticles prepared by electrospraying: Advancements in drug delivery field. J. Control. Release 2018, 286, 179-200. [CrossRef] [PubMed]

38. Bohr, A.; Yang, M.; Baldursdóttir, S.; Kristensen, J.; Dyas, M.; Stride, E.; Edirisinghe, M. Particle formation and characteristics of celecoxib-loaded poly(lactic-co-glycolic acid) microparticles prepared in different solvents using electrospraying. Polymer 2012, 53, 3220-3229. [CrossRef]

39. Massella, D.; Leone, F.; Peila, R.; Barresi, A.A.; Ferri, A. Functionalization of cotton fabrics with polycaprolactone nanoparticles for transdermal release of melatonin. J. Funct. Biomater. 2017, 9, 1. [CrossRef]

40. Council of Europe. European Pharmacopoeia, 6th ed.; Council of Europe: Strasbourg, France, 2007.

41. Xie, J.; Ng, W.J.; Lee, L.Y.; Wang, C.H. Encapsulation of protein drugs in biodegradable microparticles by co-axial electrospray. J. Colloid Interface Sci. 2008, 317, 469-476. [CrossRef]

42. Yao, J.; Kuang Lim, L.; Xie, J.; Hua, J.; Wang, C.-H. Characterization of electrospraying process for polymeric particle fabrication. J. Aerosol Sci. 2008, 39, 987-1002. [CrossRef] 
43. Gañán-Calvo, A.M.; Dávila, J.; Barrero, A. Current and droplet size in the electrospraying of liquids. Scaling laws. J. Aerosol Sci. 1997, 28, 249-275. [CrossRef]

44. Jaworek, A.; Krupa, A. Classification of the modes of ehd spraying. J. Aerosol Sci. 1999, 30, $873-893$. [CrossRef]

45. Jayasinghe, S.N.; Edirisinghe, M.J. Effect of viscosity on the size of relics produced by electrostatic atomization. J. Aerosol Sci. 2002, 33, 1379-1388. [CrossRef]

46. Zhou, F.L.; Hubbard Cristinacce, P.L.; Eichhorn, S.J.; Parker, G.J. Preparation and characterization of polycaprolactone microspheres by electrospraying. Aerosol Sci. Technol. 2016, 50, 1201-1215. [CrossRef] [PubMed]

47. Arya, N.; Chakraborty, S.; Dube, N.; Katti, D.S. Electrospraying: A facile technique for synthesis of chitosan-based micro/nanospheres for drug delivery applications. J. Biomed. Mater. Res. B Appl. Biomater. 2009, 88, 17-31. [CrossRef] [PubMed]

48. Ghayempour, S.; Mortazavi, S.M. Fabrication of micro-nanocapsules by a new electrospraying method using coaxial jets and examination of effective parameters on their production. J. Electrost. 2013, 71, 717-727. [CrossRef]

49. Zhang, L.; Huang, J.; Si, T.; Xu, R.X. Coaxial electrospray of microparticles and nanoparticles for biomedical applications. Expert Rev. Med. Devices 2012, 9, 595-612. [CrossRef] [PubMed]

50. Chang, M.W.; Stride, E.; Edirisinghe, M. Controlling the thickness of hollow polymeric microspheres prepared by electrohydrodynamic atomization. J. R. Soc. Interface 2010, 7, S451-S460. [CrossRef]

51. Wu, Y.; Clark, R.L. Controllable porous polymer particles generated by electrospraying. J. Colloid Interface Sci. 2007, 310, 529-535. [CrossRef]

52. Park, C.H.; Lee, J. Electrosprayed polymer particles: Effect of the solvent properties. J. Appl. Polym. Sci. 2009, 114, 430-437. [CrossRef]

53. Huang, X.; Gao, J.; Li, W.; Xue, H.; Li, R.K.Y.; Mai, Y.-W. Preparation of poly( $\varepsilon$-caprolactone) microspheres and fibers with controllable surface morphology. Mater. Des. 2017, 117, 298-304. [CrossRef]

54. Lee, H.; Paik, D.-H.; Jeong, K.-Y.; Gang, R.-H.; Lee, J.; Choi, S.-W. Fabrication of poly(methyl methacrylate) and $\mathrm{TiO}_{2}$ composite microspheres with controlled morphologies and porous structures by electrospraying. J. Mater. Sci. 2015, 50, 6531-6538. [CrossRef] 